\title{
Bringing the Gap between Primary Health Care and Health Economics
}

\author{
Zoi Tsimtsiou ${ }^{1,2^{*}}$ \\ ${ }^{1}$ Department of Hygiene, School of Medicine, Aristotle University of Thessaloniki, Thessaloniki, Greece \\ 2Urban Health Center of Evosmos, Thessaloniki, Greece
}

*Corresponding author: Zoi Tsimtsiou, Department of Hygiene, School of Medicine, Aristotle University of Thessaloniki, Thessaloniki, Greece, Tel: 003069770626; Email: zoitsimtsiou@yahoo.gr

Received date: May 08, 2017; Accepted date: May 10, 2017; Published date: May 12, 2017

Copyright: (c) 2017 Tsimtsiou Z. This is an open-access article distributed under the terms of the Creative Commons Attribution License, which permits unrestricted use, distribution, and reproduction in any medium, provided the original author and source are credited.

Citation: Tsimtsiou Z (2017) Bringing the Gap between Primary Health Care and Health Economics. Health Econ Outcome Res 3: e106.

\section{Editorial}

According to the World Health Report 2008: Primary Health Care (Now More Than Ever), the primary health care approach can be an efficient, fair, and cost-effective way to organize a health system [1]. It can prevent much of the disease burden, and it can also prevent people with minor complaints from flooding the emergency wards of hospitals. Primary health care's core features (person-centeredness, comprehensiveness, integration and continuity of care) have been repeatedly associated with better health outcomes and with higher user satisfaction $[2,3]$.

The indispensable need to provide efficient and equitable services from primary health care, against a background of increasing demands and limited financial resources, is an emerging challenge. Primary care physicians play an important role in the allocation of health care funds. Everyday decisions on diagnostic testing, prescribing medications, or requesting consultation from specialists have inevitable consequences in the available health care resources [4]. However, undergraduate medical education and post-graduate and continuous education for primary care physicians mainly focuses on understanding the reliability and validity of diagnostic tests and the guidelines on reaching therapeutic decisions, while minimal to no emphasis is given on the economic evaluation of their choices.

Bringing the gap between the separate worlds of primary health care and health economics could be achieved by different ways that could work in synergy. For instance, taking advantage of the electronic systems used for prescribing and showing to physicians the actual cost of every diagnostic or therapeutic choice they make in real time could help them taking the financial parameter into consideration when selecting the appropriate approach among equals. Furthermore, raising awareness on the basic concepts of economic evaluation in physicians would aim them in developing pragmatic decision-making frameworks which draw upon economic concepts and principles [4-6], while continuing to respect the uniqueness of patients in who they are applied. Educating physicians to understand the different types of economic evaluation studies (cost- effectiveness, cost-utility and costbenefit studies) and their differences in how they measure health benefits could assist in the provision of cost-effective, efficient and patient-centered primary health care services [4-6].

Taking into consideration that the current economic challenges and that inevitably poor economic growth is strongly related with stagnating resources for health, bridging the gap between primary health care and health economics is a high priority; "now more than ever". Recognizing the need to arm primary care physicians with an understanding of the basic principles of economic evaluation can be an effective way to achieve a closer relationship between health economists and those who commission and deliver primary care [5]. It can enable physicians to understand the impact of their clinical decision-making on the financial resources and take into consideration this parameter when trying to choose the best patient-centered plan among the available evidence-based practices. Taking costs into consideration should not be confused with providing lower quality primary health care. It seems to be the best way to ensure that the available resources will be used in the most efficient way, while more funds with will available to cover any future needs, always respecting the special needs of every individual patient, always "putting the patient first".

\section{References}

1. World Health Report (2008) Primary Health Care (Now More Evere).

2. O'Malley AS, Rich EC, Maccarone A, DesRoches CM, Reid RJ (2015) Disentangling the Linkage of Primary Care Features to Patient Outcomes: A Review of Current Literature, Data Sources, and Measurement Needs. J Gen Intern Med 30: 576-585.

3. Fan VS, Burman M, McDonell MB, Fihn SD (2005) Continuity of Care and Other Determinants of Patient Satisfaction with Primary Care. J Gen Intern Med 20: 226-233.

4. McBrien KA, Manns B (2013) Approach to economic evaluation in primary care: review of a useful tool for primary care reform. Can Fam Physician 59: 619-27.

5. Kernick DP (2000) The impact of health economics on healthcare delivery. A primary care perspective. Pharmacoeconomics 18: 311-315.

6. Kernick DP (2003) Introduction to health economics for the medical practitioner. Postgrad Med J 79: 147-150. 